SV

\title{
VIBRATION AND SEN SITIVITY ANALYSIS OF A SPINNING DISK UNDER TANGENTIAL EDGE LOADS
}

\author{
JEN-SAN Chen \\ Department of Mechanical Engineering, National Taiwan University, Taipei 107, Taiwan
}

(Received 10 January 1997, and in final form 28 July 1997)

\begin{abstract}
The vibration and stability of a spinning disk under space-fixed tangential edge loads are studied. Both conservative and follower loads are considered. Finite Fourier transform method is employed to discretize the equation of motion. Sensitivity analysis is then performed up to the second order to predict the effects of various Fourier components of the edge loads on the eigensolutions of the spinning disk. In the case when the tangential edge load is uniform and of the follower type, the first order derivatives of the eigenvalues are real. If the direction of the edge load is opposite to the sense of the disk rotation, then all the forward traveling modes will be destabilized, and all the backward and reflected waves will be stabilized. When the uniform edge load is conservative, the first order derivatives are always zero, while the second order derivatives are generally purely imaginary. When the conservative edge load is of the form $f_{k} \cos k \theta$, it is predicted that the modal interaction between two non-reflected waves is always of the veering type. On the other hand, the modal interaction between one non-reflected wave and one reflected wave is always of the merging type.
\end{abstract}

(C) 1998 Academic Press

\section{INTRODUCTION}

The vibration analysis of a spinning disk under space-fixed edge loads attracts attention because of its applications in such fields as wood-cutting industry, grind wheel operation, and silicon wafer slicing. Carlin and his co-workers' investigation [1] appears to be the first paper attempting to calculate the natural frequencies of a spinning disk under a concentrated radial edge load. Radcliffe and Mote [2] extended the work of Carlin et al. by considering a general concentrated edge load with both radial and tangential components. The formulations in these two papers did not take into account the effects of relative motion between the spinning disk and the space-fixed edge load. Chonan et al. [3] studied the vibration and deflection of a pre-tensioned inside-diameter saw blade used in cutting silicon crystal ingot. Because the cutting force in their application is too small compared to the membrane stresses from initial tensioning, they did not observe any significant effect on the natural frequencies and stability of the rotating disk.

Recently Chen [4] reformulated the problem with emphasis on the effects of relative motion between the disk and the radial concentrated edge load on the stability and natural frequencies of the loaded disk. He used a finite element scheme to calculate the eigenvalues of the system and reported that both divergence and flutter instabilities are induced by the space-fixed radial edge load when the disk is spinning at or beyond the first critical speed. 
Chen [5] also investigated in detail the effects of each Fourier component of the concentrated radial edge load on the natural frequencies and stability of the spinning disk.

In an independent study, Shen and Song [6] used multiple scale method to analyze the stability properties of a spinning disk under a concentrated follower edge load with both radial and tangential components. They reported that the stiffness matrix due to the asymmetric membrane stress field was Hermitian. This result implies that there exists a scalar potential corresponding to the external force, which is in contradiction to the definition of a non-conservative follower force [7]. In view of this unreasonable result, their conclusions on the effects of a tangential edge load on the stability of the spinning disk are in doubt.

Eigensolution sensitivity analysis has long been used to predict the effects of various parameters on the natural frequencies and mode shapes of structures. Chen and Bogy [8] cast the second order equation of motion of a general gyroscopic continuum into a first order form, and formulated the first order derivative of the eigenvalue with respect to various system parameters. Although in many applications, the first order derivative of the eigenvalue is sufficient, higher order sensitivity analysis is desirable in some cases for more accurate results. In particular, when the first order derivative vanishes identically, higher order derivatives will be needed to predict the effects of the load parameters. It appears difficult to extend Chen and Bogy's formulation directly to higher order derivatives due to the fact that the eigenvectors in the first order space also contain the eigenvalues. However, it will be demonstrated in this paper that higher order sensitivity analysis is possible for a special class of gyroscopic continuum in which the unperturbed eigenfunctions are orthogonal with respect to the mass, gyral, and stiffness operators.

In the current paper we first write the dimensionless equation of motion for a spinning disk under space-fixed tangential edge load with respect to the stationary coordinate system. We adopt the finite Fourier transform method as proposed by Zauderer [9] to discretize the partial differential equation. This procedure is particularly useful when the boundary conditions of the perturbed eigenfunctions are different from those of the unperturbed eigenfunctions. It will be demonstrated that the stiffness matrix is Hermitian when the edge load is conservative, and is non-Hermitian when the edge load is of the follower type. Regarding the sensitivity analysis, after finding that the first order derivatives of the eigenvalues of the spinning disk are zero when the edge load is conservative, we proceed to derive the first order derivatives of the eigenfunctions and the second order derivatives of the eigenvalues. The predictions based on the sensitivity analysis are compared to the numerical results from the finite Fourier transform method.

\section{EQUATION OF MOTION}

Figure 1 shows a spinning circular disk which is clamped at the inner radius $r^{*}=a$ and subjected to a tangential force at the outer radius $r^{*}=b$. We assume that the in-plane tangential edge traction can be expanded in a Fourier series

$$
\sum_{k=0}^{\infty} f_{k}^{*} \cos k \theta
$$

$f_{k}^{*}$ have the dimensions of stress and represent the weighting of each Fourier component. The disk is rotating with constant speed $\Omega^{*}$, while the edge load is fixed in space. The magnitude of the edge load remains the same when the disk vibrates laterally. The transverse displacement of the spinning disk with respect to the stationary coordinate system $\left(r^{*}, \theta\right)$ is denoted as $w^{*}$, and the physical time is denoted as $t^{*}$. The in-plane stress 
fields induced by the centrifugal effect are $\sigma_{r}^{*}$ and $\sigma_{\theta}^{*}$. The in-plane stress fields induced by the Fourier component $\cos k \theta$ are $\hat{\sigma}_{r(k)}^{*}, \hat{\sigma}_{\theta(k)}^{*}$, and $\hat{\sigma}_{r \theta}^{*}$, and can be expressed as

$$
\begin{gathered}
\hat{\sigma}_{r(k)}^{*}\left(r^{*}, \theta\right)=\tilde{\sigma}_{r(k)}^{*}\left(r^{*}\right) \sin k \theta \\
\hat{\sigma}_{\theta(k)}^{*}\left(r^{*}, \theta\right)=\tilde{\sigma}_{\theta(k)}^{*}\left(r^{*}\right) \sin k \theta \quad k=0,1,2, \ldots \\
\hat{\sigma}_{r(k)}^{*}\left(r^{*}, \theta\right)=\tilde{\sigma}_{r(\theta)}^{*}\left(r^{*}\right) \cos k \theta
\end{gathered}
$$

$\hat{\sigma}_{r(k)}^{*}, \hat{\sigma}_{\theta(k)}^{*}$, and $\hat{\sigma}_{r(t)}^{*}$ are functions of radial coordinate $r^{*}$ only, and can be found readily in [10].

It is convenient to introduce some dimensionless variables

$$
w=\frac{w^{*}}{h}, \quad r=\frac{r^{*}}{b}, \quad \eta=\frac{a}{b}, \quad t=\frac{t^{*}}{b^{2}} \sqrt{\frac{D}{\rho h}}, \quad \Omega=\Omega^{*} b^{2} \sqrt{\frac{\rho h}{D}},
$$

where $\rho, h$, and $D$ are the mass density, thickness, and flexural rigidity of the disk, respectively. The stresses are also nondimensionalized as follows,

$$
\left(\sigma_{r}, \sigma_{\theta}, \tilde{\sigma}_{r}, \tilde{\sigma}_{\theta}, \tilde{\sigma}_{r \theta}, f_{k}\right)=\frac{h b^{2}}{D}\left(\sigma_{r}^{*}, \sigma_{\theta}^{*}, \tilde{\sigma}_{r}^{*}, \tilde{\sigma}_{\theta}^{*}, \tilde{\sigma}_{r \theta}^{*}, f_{k}^{*}\right) .
$$

We define the dimensionless membrane operator $L$ associated with the axisymmetrical stress field due to the centrifugal force, and the membrane operator $\hat{L}_{(k)}$ associated with the stress field due to the Fourier component $\cos k \theta$ of the edge load as

$$
L \equiv-\frac{1}{r}\left[\frac{\partial}{\partial r}\left(r \sigma_{r} \frac{\partial}{\partial r}\right)+\frac{\partial}{\partial \theta}\left(\frac{1}{r} \sigma_{\theta} \frac{\partial}{\partial \theta}\right)\right]
$$

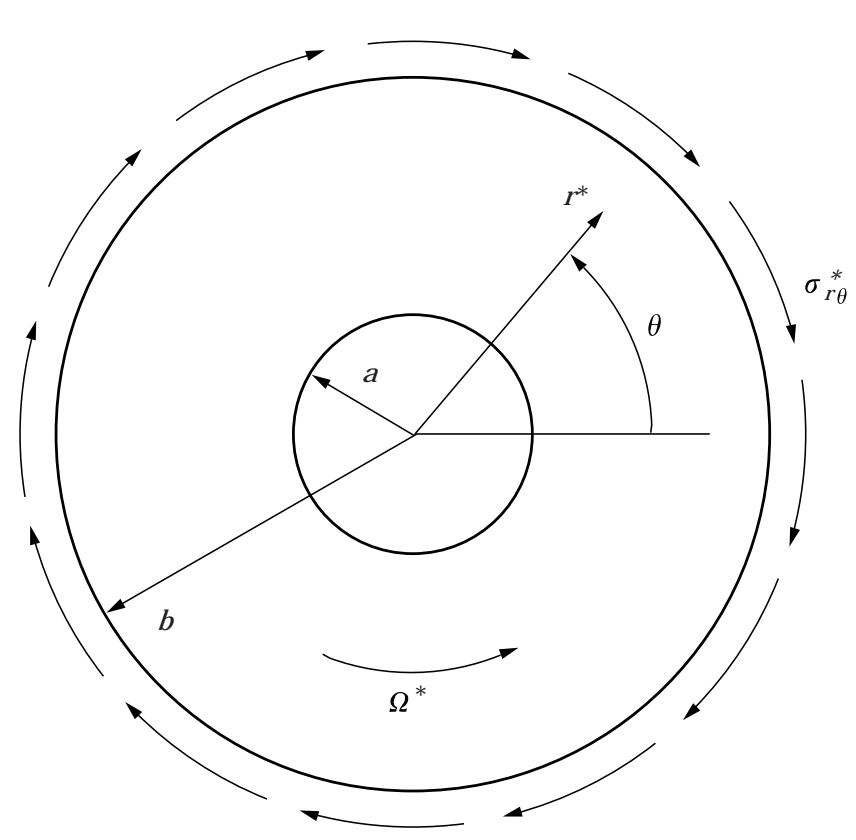

Figure 1. A spinning disk under space-fixed tangential edge load. 


$$
\begin{aligned}
\hat{L}_{(k)} & \equiv-\frac{1}{r}\left[\frac{\partial}{\partial r}\left(r \tilde{\sigma}_{r(k)} \sin k \theta \frac{\partial}{\partial r}+\tilde{\sigma}_{r \theta(k)} \cos k \theta \frac{\partial}{\partial \theta}\right)\right. \\
& \left.+\frac{\partial}{\partial \theta}\left(\tilde{\sigma}_{r \theta(k)} \cos k \theta \frac{\partial}{\partial r}+\frac{1}{r} \tilde{\sigma}_{\theta(k)} \sin k \theta \frac{\partial}{\partial \theta}\right)\right] .
\end{aligned}
$$

It is noted that

$$
\tilde{\sigma}_{r(k)}(1)=0 \quad \text { and } \quad \tilde{\sigma}_{r \theta(k)}(1)=-1
$$

due to the in-plane traction boundary conditions on the outer rim.

The equation of motion in terms of these dimensionless variables and operators can be written as follows:

$$
w_{, t t}+G w_{, t}+(K+\hat{K}) w=0
$$

where

$$
G \equiv 2 \Omega \frac{\partial}{\partial \theta}, \quad K \equiv \Omega^{2} \frac{\partial^{2}}{\partial \theta^{2}}+\nabla^{4}+L, \quad \hat{K} \equiv \sum_{k=0}^{\infty} f_{k} \hat{L}_{(k)}
$$

The dimensionless boundary conditions for equation (5) at the inner radius are

$$
w=w_{, r}=0 \quad \text { at } \quad r=\eta .
$$

At the outer radius we consider two types of edge loads. For a conservative load, the direction remains the same when the disk vibrates laterally. The boundary conditions corresponding to this type of load are

$$
B_{1} w+\sum_{k=0}^{\infty} f_{k} \hat{B}_{1(k)} w=B_{2} w=0 \quad \text { at } \quad r=1
$$

where the boundary operators are defined as

$$
\begin{gathered}
B_{1} \equiv \frac{\partial}{\partial r}\left(\frac{\partial^{2}}{\partial r^{2}}+\frac{\partial}{r \partial r}+\frac{\partial^{2}}{r^{2} \partial \theta^{2}}\right)+\frac{1-v}{r^{2}}\left(\frac{\partial^{3}}{\partial r \partial \theta^{2}}-\frac{\partial^{2}}{r \partial \theta^{2}}\right) \\
\hat{B}_{1(k)} \equiv \cos (k \theta) \frac{\partial}{r \partial \theta}, \quad B_{2} \equiv \frac{\partial^{2}}{\partial r^{2}}+\frac{v}{r}\left(\frac{\partial}{\partial r}+\frac{\partial^{2}}{r \partial \theta^{2}}\right)
\end{gathered}
$$

where $v$ is the Poisson ratio. On the other hand, for a follower force whose direction is always tangent to the circumferential slope of the vibrating disk, the boundary conditions are

$$
B_{1} w=B_{2} w=0 \quad \text { at } \quad r=1 .
$$

For a freely spinning disk, $\hat{K} \equiv 0$ in equation (5), and the eigenvalues are purely imaginary, i.e. $\lambda_{m n}^{0}=i \omega_{m n}^{0}$, where $\omega_{m n}^{0}$ is real. The eigenfunction corresponding to $\lambda_{m n}^{0}$ is in general complex and assumes the following separable form

$$
w_{m n}^{0}=R_{m n}(r) \mathrm{e}^{i n \theta}
$$


$w_{m n}^{0}$ in equation (11) with positive $n$ and positive $\omega_{m n}$ is a backward travelling wave with $n$ nodal diameters and $m$ nodal circles, which is also denoted by $(m, n)_{b}$. Similarly, $w_{m n}^{0}$ with negative $n$ and positive $\omega_{m n}^{0}$ is a forward travelling wave $(m,-n)_{f}$. The real-valued functions $R_{m n}$ for $(m,-n)_{f}$ and $(m, n)_{b}$ are the same. The critical speed $\Omega_{c}$ for the mode $(m, n)$ is defined as the rotation speed at which $\omega_{m n}$ of the backward travelling wave $(m, n)_{b}$ becomes zero. For $\Omega$ greater than $\Omega_{c}$, the backward travelling mode appears to travel forward, and is called a "reflected wave", denoted by $(m, n)_{r}$. It is noted that for a reflected wave, the integer $n$ is considered as positive, while the natural frequency $\omega_{m n}^{0}$ is considered as negative.

We define the inner product between two functions as

$$
\left\langle w_{m n}^{0}, w_{p q}^{0}\right\rangle=\int_{0}^{2 \pi} \int_{\eta}^{1} \bar{w}_{m n}^{0} w_{p q}^{0} r \mathrm{~d} r \mathrm{~d} \theta,
$$

where the overbar means complex conjugate. It can be readily shown that the eigenfunctions are orthogonal with respect to the operators $G$ and $K$ in the above inner product space. In particular, the mode $(m,-n)_{f}$ is orthogonal to $(m, n)_{b}$ in the sub-critical speed range, and is orthogonal to $(m, n)_{r}$ in the super-critical speed range. Consequently, we can normalize the eigenfunctions in the manner.

$$
\left\langle w_{m n}^{0}, w_{m n}^{0}\right\rangle=1 .
$$

\section{DISCRETIZATION}

We express the solution $w$ of equation (5) as a series expansion of $w_{m n}^{0}$ in equation (11),

$$
w(r, \theta, t)=\sum_{m=0}^{\infty} \sum_{n=-\infty}^{\infty}\left[c_{m n}(t) w_{m n}^{0}(r, \theta)\right]
$$

The coefficients $c_{m n}(t)$ are generally complex functions of time, and can be obtained as

$$
c_{m n}=\left\langle w_{m n}^{0}, w\right\rangle
$$

Since the boundary conditions for $w_{m n}$ and $w$ may be different, the series (14) may not be differentiable term by term. Therefore we cannot substitute the series directly into equation (5). Instead, we multiply equation (5) by $\bar{w}_{p q}^{0}$ and integrate over the annular region to obtain

$$
\ddot{c}_{p q}+\left\langle w_{p q}^{0}, G_{w, t}+(K+\hat{K}) w\right\rangle=0 .
$$

After integrating by parts and employing relation (15), we find that

$$
\begin{gathered}
\left\langle w_{p q}^{0}, G w_{, t}\right\rangle=2 i q \Omega \dot{c}_{p q} \\
\left\langle w_{p q}^{0}, K w\right\rangle=\left\langle K w_{p q}^{0}, w\right\rangle-\int_{0}^{2 \pi}\left[r \bar{w}_{p q}^{0} \sum_{k=0}^{\infty} f_{k} \hat{B}_{1(k)} w\right]_{r=1} \mathrm{~d} \theta .
\end{gathered}
$$

For a follower edge load, the boundary integral in equation (18) is zero because $\hat{B}_{1(k)} \equiv 0$, and

$$
\left\langle w_{p q}^{0}, K w\right\rangle=\omega_{p q}^{0}\left(\omega_{p q}^{0}+2 q \Omega\right) c_{p q} .
$$


On the other hand, for a conservative edge load, further integration by part yields

$$
\left\langle w_{p q}^{0}, K w\right\rangle=\omega_{p q}^{0}\left(\omega_{p q}^{0}+2 q \Omega\right) c_{p q}-\sum_{k=0}^{\infty} \sum_{m=0}^{\infty} \sum_{\substack{n=-\infty \\ n-q= \pm k}}^{\infty} i f_{k} \alpha_{k} q R_{p q}(1) R_{m n}(1) c_{m n}
$$

where $\alpha_{0}=1$, and $\alpha_{k}=1 / 2$ when $k \neq 0$. The last inner product in equation (16) can also be integrated by parts, with use of the boundary conditions (4), to obtain

$$
\left\langle w_{p q}^{0}, \hat{K} w\right\rangle=\sum_{k=0}^{\infty} \sum_{m=0}^{\infty} \sum_{\substack{n=-\infty \\ n-q= \pm k}}^{\infty} f_{k} c_{m n}\left\{S_{m n(k)}^{p q}+i \alpha_{k} q R_{p q}(1) R_{m n}(1)\right\}
$$

where

$$
\begin{aligned}
S_{m n(k)}^{p q} & =i \alpha_{k} \int_{\eta}^{1}\left[\mp r \tilde{\sigma}_{r(k)} R_{p q, r} R_{m n, r}+n \tilde{\sigma}_{r \theta(k)} R_{p q, r} R_{m n}-q \tilde{\sigma}_{r \theta(k)} R_{p q} R_{m n, r}\right. \\
& \left.\mp n q \frac{\tilde{\sigma}_{\theta(k)}}{r} R_{p q} R_{m n}\right] \mathrm{d} r .
\end{aligned}
$$

It is noted that in the triple summation in equations (20) and (21), only those modes with the number of nodal diameters $n=q \pm k$ will participate in the summation. The $\mp$ sign in equation (22) corresponds to the \pm sign in the relation $n-q= \pm k$. The radial function $R_{m n}(r)$ of the disk spinning at a particular speed can be either calculated by a power series method [11], or by an independent finite element scheme [4]. The integral in equation (22) is performed numerically. It is also noted that $S_{m n(k)}^{p q}$ is Hermitian, i.e. $S_{m n(k)}^{p q}=\bar{S}_{p q(k)}^{m n}$. In summary, the discretized matrix equation can be written as

$$
\ddot{c}_{p q}+2 i q \Omega \dot{c}_{p q}+\omega_{p q}^{0}\left(\omega_{p q}^{0}+2 q \Omega\right) c_{p q}+\sum_{k=0}^{\infty} \sum_{m=0}^{\infty} \sum_{\substack{n=-\infty \\ n-q= \pm k}}^{\infty} f_{k} \hat{K}_{m n(k)}^{p q} c_{m n}=0
$$

where $\hat{K}_{m n(k)}^{p q}$ is non-Hermitian for a follower edge load

$$
\hat{K}_{m n(k)}^{p q}=S_{m n(k)}^{p q}+i \alpha_{k} q R_{p q}(1) R_{m n}(1)
$$

and is Hermitian for a conservative edge load

$$
\hat{K}_{m n(k)}^{p q}=S_{m n(k)}^{p q} \text {. }
$$

The matrix equation (23) can be cast in the first order form, and then solved for its eigensolutions by any eigensolver, such as Eispack.

The solid lines in Figures 2 and 3 show the relations between the eigenvalues of the spinning disk and the rotation speed when the disk is under follower edge loads $f_{0}=30$ and $f_{4}=60$, respectively. The ordinate of the lower graph is the absolute value of the natural frequency, and the ordinate of the upper graph is the real part of the eigenvalue. The abscissa is the rotation speed. The clamping ratio $\eta$ and the Poisson ratio $v$ are chosen as 0.5 and $0 \cdot 27$, respectively. Only the modes with less than five nodal diameters and six nodal circles are included in the calculations. The dashed lines represent the frequency loci of a freely spinning disk. It is found that when the direction of the uniform follower edge load is opposite to the sense of the disk rotation, it will destabilize all the forward travelling 
SPINNING DISK
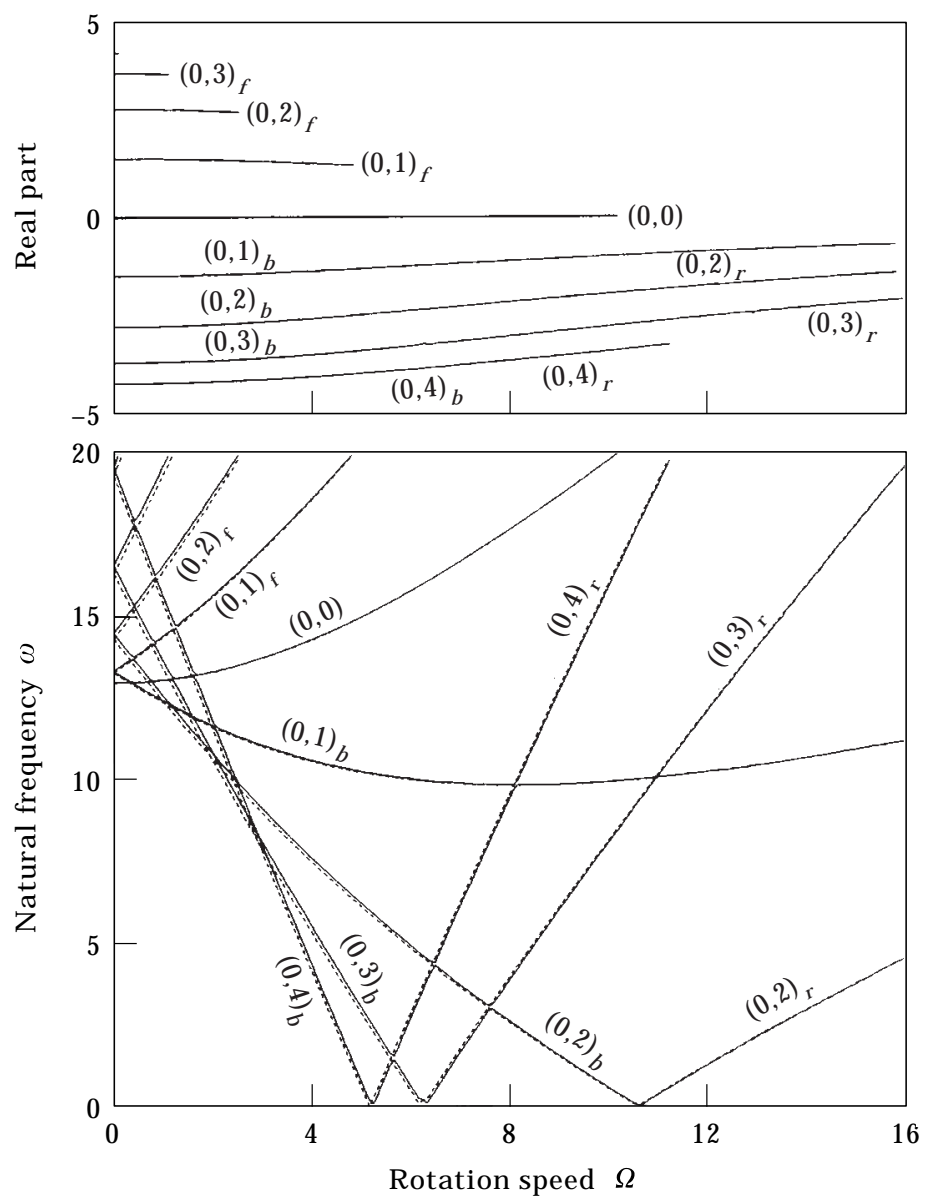

Figure 2. Relations between the eigenvalues and the rotation speed when the disk is under uniform follower edge load $f_{0}=30$.

modes and stabilize all the backward and reflected waves. It has negligible effect on the natural frequencies of the spinning disk. These effects are completely different from its radial counterpart. A uniform radial edge load affects only the natural frequencies but not the stability properties of the spinning disk. In the case of non-uniform follower load, Figure 3 shows that flutter instability is induced in the subcritical speed range when frequency locus of $(0,0)$ meets $(0,4)_{b}$, and $(0,1)_{f}$ meets $(0,3)_{b}$. Divergence instability is induced in the neighborhood of the critical speed of mode $(0,2)$. Frequency loci veering occurs as mode $(0,3)_{r}$ meets $(0,1)_{b}$.

Figure 4 shows the effects of uniform conservative edge load $f_{0}=80$ on the natural frequencies of the spinning disk. The natural frequencies of all zero-nodal-circle modes decrease, and no instability or modal interaction is induced. Figure 5 shows the effect of non-uniform conservative edge load $f_{4}=60$ on the eigenvalues of the system. It is interesting to compare Figures 3 and 5 in which the types of modal interactions change as the edge load changes from the follower type to the conservative type. In the case of conservative edge load, the effects of the tangential component are similar to the effects of its radial counterpart [5]. 


\section{EIGENSOLUTION SENSITIVITY}

\subsection{FIRST ORDER DERIVATIVES OF EIGENVALUES}

In this section we derive the expressions for the derivatives of eigenvalues and eigenfunctions of the spinning disk with respect to various edge load coefficients $f_{k}$. First of all, we write the equations for the eigensolutions of a freely spinning disk and a spinning disk under tangential edge loads as

$$
\begin{gathered}
\left(\lambda_{m n}^{0}\right)^{2} w_{m n}^{0}+\lambda_{m n}^{0} G w_{m n}^{0}+K w_{m n}^{0}=0 \\
\lambda_{m n}^{2} w_{m n}+\lambda_{m n} G w_{m n}+(K+\hat{K}) w_{m n}=0 .
\end{gathered}
$$

The subscript $m n$ in equation (27) means that the perturbed eigenvalue $\lambda_{m n}$ and eigenfunction $w_{m n}$ will return to $\lambda_{m n}^{0}$ and $w_{m n}^{0}$ of a freely spinning disk as the tangential edge load approaches zero. The boundary conditions for equation (26) are equations (6) and (10). The boundary conditions for equation (27) are equations (6) and (7) for a conservative edge load, and are equations (6) and (10) for a follower edge load.
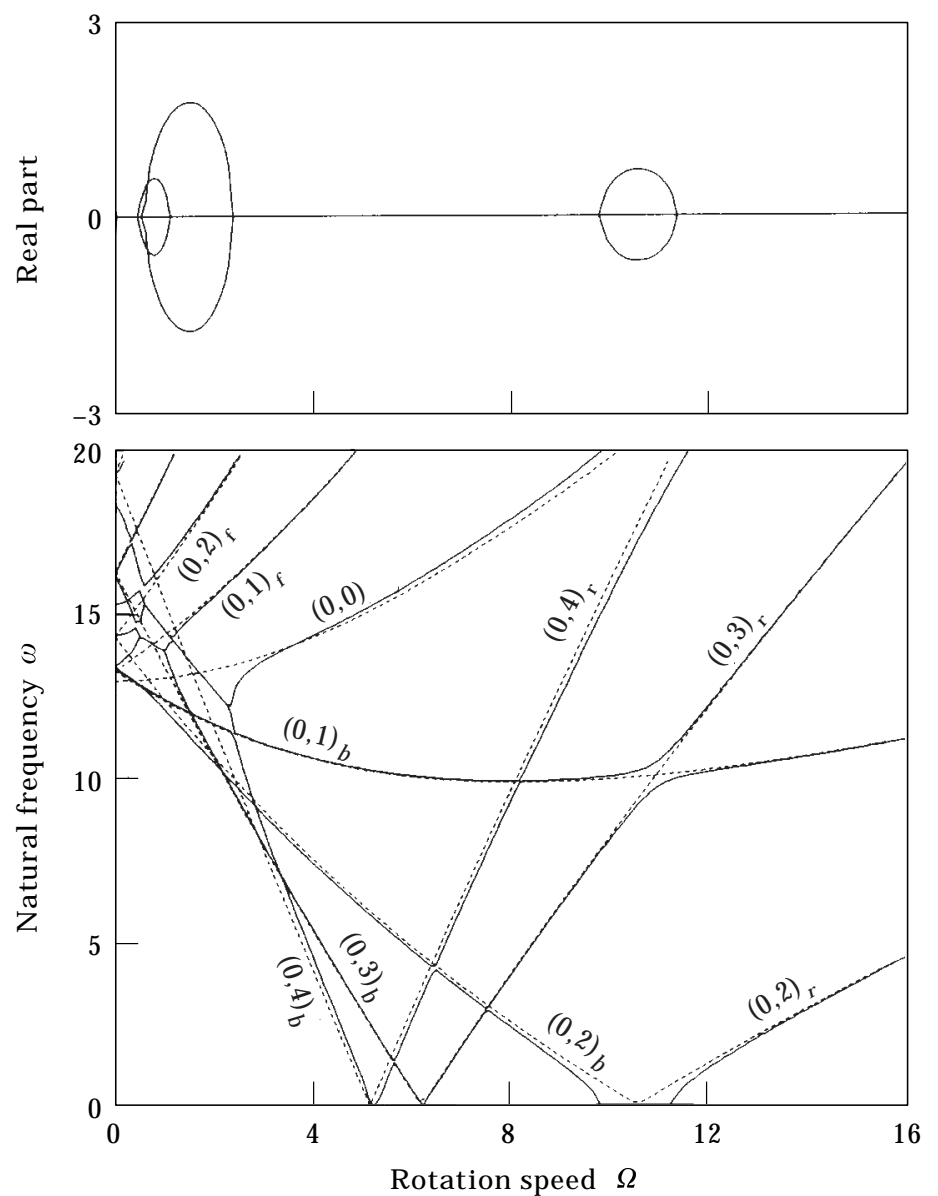

Figure 3. Relations between the eigenvalues and the rotation speed when the disk is under uniform follower edge load $60 \cos 4 \theta$. 


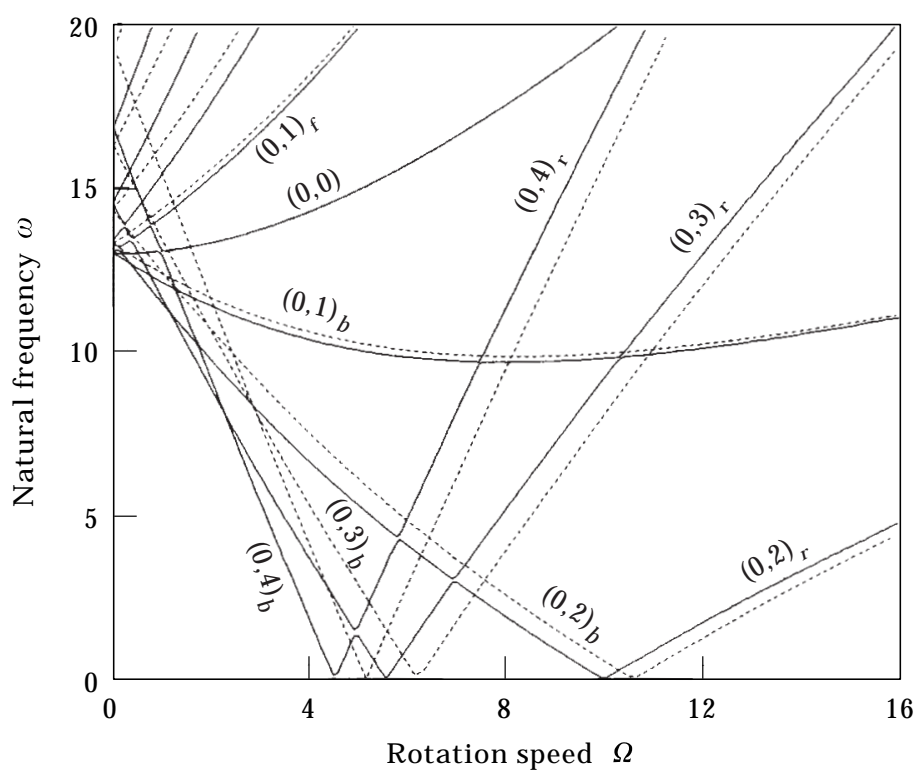

Figure 4. Relations between the eigenvalues and the rotation speed when the disk is under uniform conservative edge load $f_{0}=80$.

For a conservative edge load, we differentiate equation (27) and the boundary conditions (6) and (7), and then set, $f_{k}=0$ to obtain

$$
\begin{gathered}
2 \lambda_{m n}^{0} \lambda_{m n, k} w_{m n}^{0}+\left(\lambda_{m n}^{0}\right)^{2} w_{m n, k}+\lambda_{m n, k} G w_{m n}^{0}+\lambda_{m n}^{0} G w_{m n, k}+K w_{m n, k}+\hat{L}_{(k)} w_{m n}^{0}=0 \\
w_{m n, k}=0, \quad w_{m n, r k}=0 \quad \text { at } \quad r=\eta \\
B_{1} w_{m n, k}=-\hat{B}_{1} w_{m n}^{0}, \quad B_{2} w_{m n, k}=0 \quad \text { at } \quad r=1
\end{gathered}
$$

where the subscript $k$ represents the edge load coefficient $f_{k}$. For a follower edge load, the term associated with $\hat{B}_{1}$ in equation (30) disappears. We multiply the conjugate of equation (26) by $w_{m n, k}$, and multiply equation (28) by $\bar{w}_{m n}^{0}$, subtract these two equations and integrate over the annular region to obtain

$$
2 i\left(\omega_{m n}^{0}+n \Omega\right) \lambda_{m n, k}+\left\langle w_{m n}^{0}, K w_{m n, k}\right\rangle-\left\langle K w_{m n}^{0}, w_{m n, k}\right\rangle+\left\langle w_{m n}^{0}, \hat{L}_{(k)} w_{m n}\right\rangle=0 .
$$

By noting that the boundary conditions for $w_{m n}^{0}$ and $w_{m n, k}$ are different for a conservative edge load, and using the relations similar to equations (18) and (21) we can obtain

$$
\left.\lambda_{m n, k}\right|_{f_{k}=0}=0 .
$$

Therefore, the conservative edge load has not effect on the eigenvalues of the spinning disk to the first order.

In the case of follower edge loads, on the other hand, $\lambda_{m n, k}$ also vanishes for $k \neq 0$. For uniform edge load $f_{0}$, we obtain

$$
\left.\lambda_{m n, 0}\right|_{f_{0}=0}=-\frac{\left\langle w_{m n}^{0}, \hat{L}_{(0)} w_{m n}^{0}\right\rangle}{2 i\left(\omega_{m n}^{0}+n \Omega\right)}=-\frac{n R_{m n}^{2}(1)}{2\left(\omega_{m n}^{0}+n \Omega\right)} .
$$


It is noted that the term $\omega_{m n}^{0}+n \Omega$ is the denominator is always positive. Since this derivative is real, we can conclude that the uniform tangential follower edge load always destabilizes the forward travelling waves, and stabilizes the backward and reflected waves. As the direction of the uniform edge load reverses, its effect on the stability of the spinning disk is reversed. It is noted that the first order derivatives (32) and (33) can also be obtained by using only one-mode approximation in equation (23). The above analyses agree with the numerical results presented in Figure 2.

\subsection{FIRST ORDER DERIVATIVES OF EIGENFUNCTIONS}

In order to find the derivatives of the eigenfunctions, we express $w_{m n, k}$ in terms of the following expansion

$$
\left.w_{m n, k}\right|_{f_{k}=0}=\sum_{m=0}^{\infty} \sum_{n=-\infty}^{\infty}\left[c_{m n} w_{m n}^{0}(r, \theta)\right] .
$$
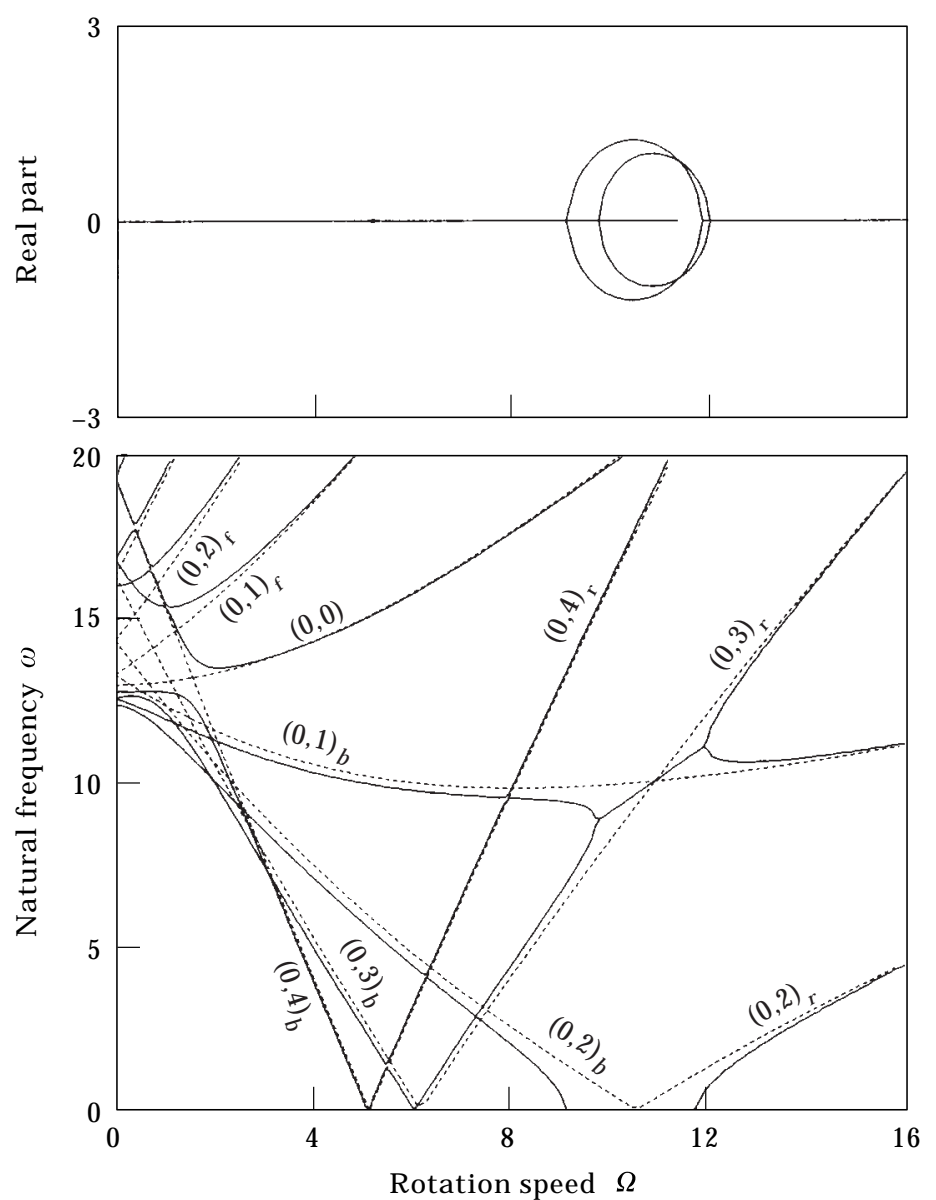

Figure 5. Relations between the eigenvalues and the rotation speed when the disk is under conservative edge load $60 \cos 4 \theta$. 
After multiplying equation (28) by $\bar{w}_{p q}^{0}$ and integrating over the annular region we obtain when $q-n= \pm k$ and $(p, q) \neq(m, n)$,

$$
c_{p q}=\frac{-\hat{K}_{m n(k)}^{p q}}{\left(\omega_{p q}^{0}-\omega_{m n}^{0}\right)\left(\omega_{p q}^{0}+\omega_{m n}^{0}+2 q \Omega\right)} .
$$

Recall that at a certain rotation speed there are in general two modes with $m$ nodal circles and $n$ nodal diameters. In the sub-critical speed range, one is a forward mode and the other is a backward mode. In the super-critical speed range, one is a forward mode and the other is a reflected mode. In any case, if $\omega_{p q}$ is the natural frequency of one $(p, q)$ mode, $\left(\omega_{p q}+2 q \Omega\right)$ is the natural frequency of the other $(p, q)$ mode, also denoted by $\omega_{p \bar{q}}$. Therefore, we can write

$$
\left.w_{m n, k}\right|_{f_{k}=0}=\sum_{\substack{p=0 \\(p, q) \pm(m, n) \\ n-q= \pm k}}^{\infty} \sum_{\substack{q=-\infty \\ n= \pm}}^{\infty} \frac{-\hat{K}_{p q(k)}^{m n} w_{m n}^{0}}{\left(\omega_{p q}^{0}-\omega_{m n}^{0}\right)\left(\omega_{p \bar{q}}^{0}+\omega_{m n}^{0}\right)} .
$$

Equation (36) is true for both conservative and follower edge loads.

\subsection{SECOND ORDER DERIVATIVES OF EIGENVALUES}

In order to find the second order derivatives of the eigenvalues, we differentiate equation (27) and boundary conditions (6) and (7) twice for a conservative edge load, and then set $f_{k}=0$ to obtain

$$
\begin{gathered}
2 \lambda_{m n}^{0} \lambda_{m n, k k} w_{m n}^{0}+2\left(\lambda_{m n, k}\right)^{2} w_{m n}^{0}+\left(\lambda_{m n}^{0}\right)^{2} w_{m n, k}+4 \lambda_{m n}^{0} \lambda_{m n, k} w_{m n, k}^{0} \\
+\lambda_{m n, k k} G w_{m n}^{0}+2 \lambda_{m n, k} G w_{m n, k}+\lambda_{m n}^{0} G w_{m n, k k}+K w_{m n, k k}+2 \hat{L}_{(k)} w_{m n, k}=0 \\
w_{m n, k k}=0, \quad w_{m n, r k k}=0 \quad \text { at } \quad r=\eta \\
B_{1} w_{m n, k k}=-2 \hat{B}_{1} w_{m n, k}, \quad B_{2} w_{m n, k k}=0 \quad \text { at } \quad r=1 .
\end{gathered}
$$

For a follower edge load, the term associated with $\hat{B}_{1}$ in equation (39) disappears. We multiply the conjugate of equation (26) by $w_{m n, k k}$, and equation (37) by $\bar{w}_{m n}^{0}$, subtract, integrate by parts over the annular region, and then use equation (36) to obtain

$$
\left.\lambda_{m n, k k}\right|_{f_{k}=0}=\sum_{\substack{p=0 \\(n, q) \pm(m, n) \\ n-q= \pm k}}^{\infty} \sum_{\substack{q=-\infty \\\left(\omega_{m n}\right.}}^{\infty} \frac{-\left(\lambda_{m n, k}||_{f_{k}=0}\right)^{2}-i \hat{K}_{p q(k)}^{m n} \hat{K}_{m n(k)}^{p q}}{\left(\omega_{m n}^{0}+n \Omega\right)\left(\omega_{p q}^{0}-\omega_{m n}^{0}\right)\left(\omega_{p \bar{q}}^{0}+\omega_{m n}^{0}\right)} .
$$

In the above derivation, we have used the relation $\left\langle w_{m n}^{0}, w_{m n, k}\right\rangle=0$, i.e. the derivative of the eigenfunction is orthogonal to the unperturbed eigenfunction.

For a conservative edge load $\left.\lambda_{m n, k}\right|_{f_{k}=0}=0$, and the term $\hat{K}_{p q(k)}^{m n} \hat{K}_{m n(k)}^{p q}$ in the numerator of equation (40) is always positive and can be replaced by $\left|\hat{K}_{m n(k)}^{p q}\right|^{2}$. For a specific component $f_{k}$, only those modes $(p, q)$ with $n-q= \pm k$ will affect the natural frequency $\omega_{m n}$. Among those modes which affect $\omega_{m n}$, the one with frequency $\omega_{p q}^{0}$ closest to $\omega_{m n}^{0}$, or $\omega_{p \bar{q}}^{0}$ closest to $-\omega_{m n}^{0}$ will be dominant in the series (40). If both modes $(m, n)$ and $(p, q)$ are in the sub-critical speed range, then $\omega_{m n}^{0}, \omega_{p q}^{0}$, and $\omega_{p \bar{q}}^{0}$ are all positive. The dominant factor in the denominator of equation (40) is $\omega_{p q}^{0}-\omega_{m n}^{0}$. By inspecting equation (40) we can see that the mode with higher natural frequency will have a positive second order derivative, and the one with lower natural frequency will have a negative second order derivative. Consequently, the natural frequencies of these two modes will be separated more as the 


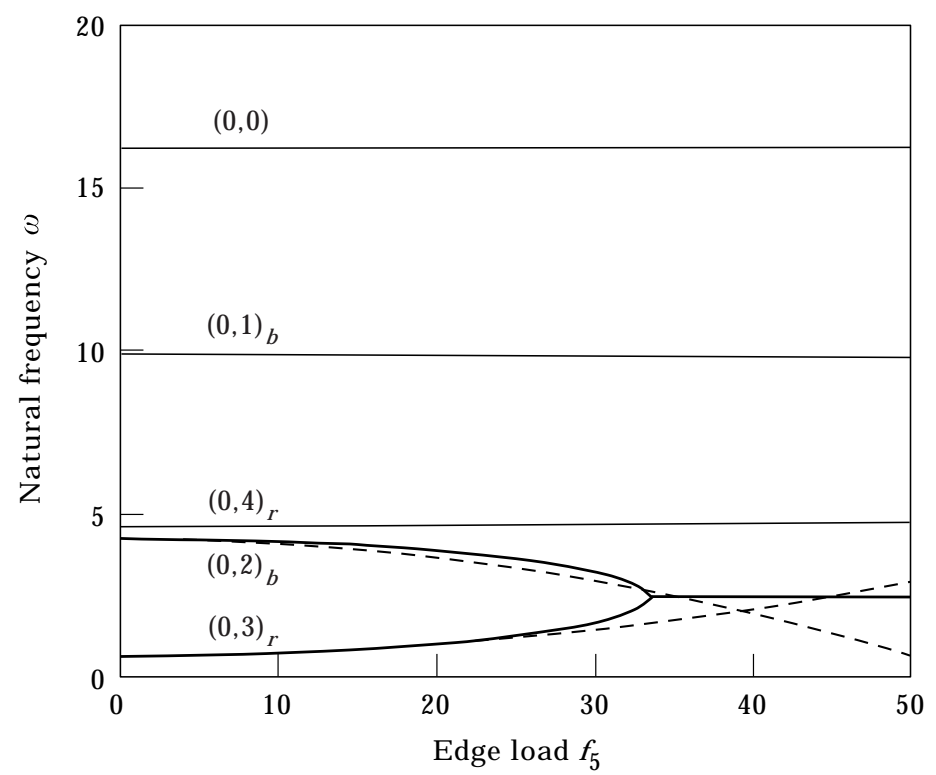

Figure 6. Relations between the eigenvalues and conservative edge load $f_{5} \cos 5 \theta$ as the disk rotates at $\Omega=6 \cdot 5$.

tangential edge load is applied. This predicts that the modal interaction of two non-reflected modes is always of the veering type.

On the other hand, if one of these two modes is a backward wave, say, $(m, n)$, and the other mode $(p, q)$ is in its super-critical speed range, then $\omega_{m n}^{0}$ and $\omega_{p q}^{0}$ are positive, and $\omega_{p \bar{q}}^{0}$ is negative. In such a case $\omega_{p q}^{0}-\omega_{m n}^{0}$ is positive and the dominant factor in equation (40) is $\omega_{p \bar{q}}^{0}+\omega_{m n}^{0}$. By inspecting equation (40) and following similar argument as in the preceding paragraph we can conclude that the second order derivatives of the eigenvalues of the modes $(m, n)_{b}$ and $(p, q)_{r}$ are both negative. Consequently, if we plot the absolute values of the natural frequencies against $f_{k}$, we can predict that the natural frequency loci of the backward mode and the reflected mode will tend to approach each other and eventually merge as $f_{k}$ increases.

Figure 6 shows the relations between the absolute values of the natural frequencies and the conservative tangential edge load $f_{5} \cos 5 \theta$ as the disk rotates at $\Omega=6 \cdot 5$. The solid lines represent the calculated results from the matrix equation (23), and the dashed lines represent the second order approximation based on equation (40). It is observed that while the second order approximation cannot reproduce the merging phenomenon, it predicts the initial curvature of the frequency loci quite well.

In the special case when the edge load is uniform, i.e. $k=0$, only those modes with the same number of nodal diameters will affect each other. Equation (40) is then reduced to

$$
\left.\lambda_{m n, 00}\right|_{f_{0}=0}=\sum_{\substack{p=0 \\ p \neq m}}^{\infty} \frac{-i\left|\hat{K}_{p n(0)}^{m n}\right|^{2}}{\left(\omega_{m n}^{0}+n \Omega\right)\left(\omega_{p n}^{0}-\omega_{m n}^{0}\right)\left(\omega_{p n}^{0}+\omega_{m n}^{0}+2 n \Omega\right)} .
$$

For the axisymmetric modes $(n=0)$, it can be seen from equations (22) and (24) that $\hat{K}_{m 0(0)}^{p 0}=0$. Therefore, the second order derivative of the natural frequency of an axisymmetric mode under conservative uniform tangential edge load is zero. For the modes with $n \neq 0$, the second and the third parentheses in the denominator of equation (41) represent the difference and the sum, respectively, of the natural frequencies of modes $(p, n)$ 
and $(m, n)$ as viewed by an observer rotating along with the disk. Furthermore, the term $\omega_{m n}^{0}+n \Omega$ represents the natural frequency of the mode $(m, n)$ as viewed by the rotating observer. Therefore, equation (41) can also be written as

$$
\left.\lambda_{m n, 00}\right|_{f_{0}=0}=\sum_{\substack{p=0 \\ p \neq m}}^{\infty} \frac{-i\left|\hat{K}_{p n(0)}^{m n}\right|^{2}}{\omega_{m n}^{0^{*}}\left[\left(\omega_{p n}^{0^{*}}\right)-\left(\omega_{m n}^{0^{*}}\right)^{2}\right]} .
$$

where the superscript $(*)$ indicates that the natural frequencies are viewed by a rotating observer. The same result can also be obtained by writing the equation of motion with respect to a frame rotating with the disk, and then by applying similar sensitivity analysis as described in this section. The resulted equation is non-gyroscopic due to the fact that uniform edge load does not present temporal variation on the boundary conditions with respect to the rotating observer. This is not the case for non-uniform edge loads. With this observation in mind and by noting that $\hat{K}_{p(-n)(0)}^{m(-n)}=\hat{K}_{p n(0)}^{m n}$, we can predict that to a space-fixed observer, $\lambda_{m n, 00}$ should be the same for the forward mode $(m,-n)_{f}$ and the backward mode $(m, n)_{b}$.

Figure 7 shows the relations between the absolute values of the natural frequencies and the uniform conservative tangential edge load $f_{0}$ as the disk rotates at $\Omega=8$. It is observed that the edge load tends to increase the natural frequencies of the reflected modes $(0,3)$, and $(0,4)_{r}$, and tends to decrease the natural frequencies of the forward and backward modes with zero nodal circle. The natural frequency of $(0,0)$ mode is not affected by the uniform edge load. The natural frequency loci of modes $(0,3)_{r}$ and $(0,3)_{f}$, and $(0,4)_{r}$ and $(0,4)_{f}$, merge eventually and flutter instability is then induced with flutter frequencies equal to $3 \Omega$ and $4 \Omega$ respectively. The natural frequency of mode $(0,2)_{b}$ decreases down to zero as $f_{0}$ increases, and then reflects back to merge with the natural frequency of $(0,2)_{f}$. The flutter frequency of the $(0,2)$ mode is $2 \Omega$. These phenomena can also be predicted by an observer rotating with the disk. To an observer rotating with the disk, all the natural frequencies of

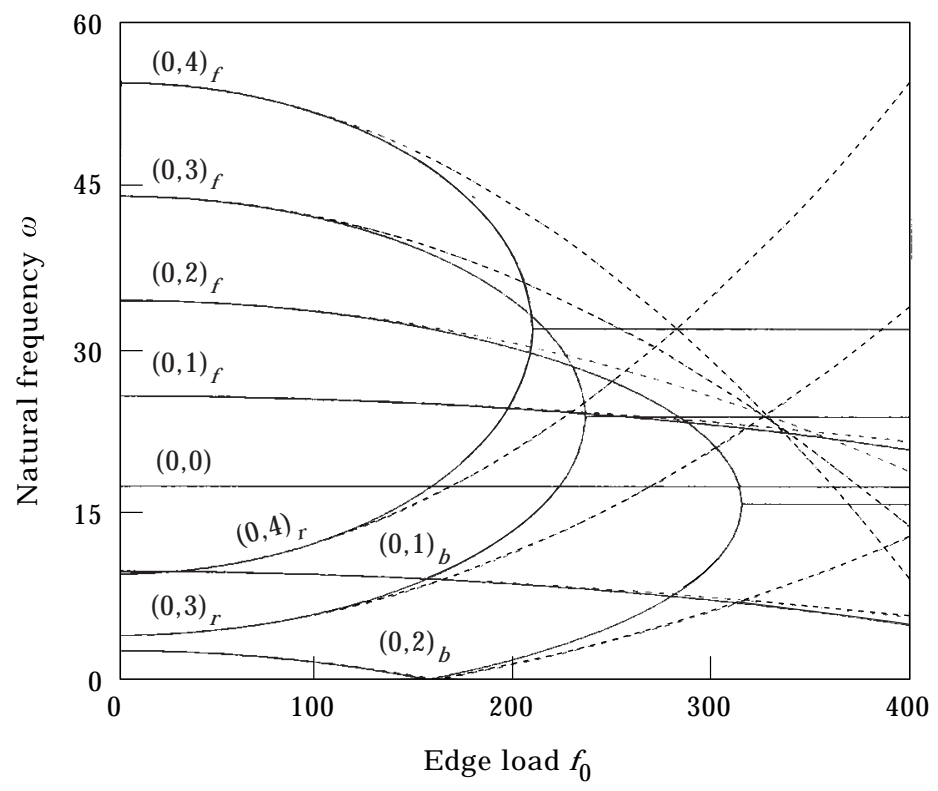

Figure 7. Relations between the eigenvalues and conservative edge load $f_{0}$ as the disk rotates at $\Omega=8$. 
the zero-nodal-circle modes decrease eventually to zero, and divergence instability is then induced. The divergence instability to a rotating observer is equivalent to the flutter instability with frequency equal to $n \Omega$ to a space-fixed observer.

\section{DISCUSSIONS AND CONCLUSIONS}

The finite Fourier transform method is employed to discretize the partial differential equation of a spinning disk under space-fixed tangential edge loads. This method is mathematically rigorous and is especially useful in treating the continuous system whose boundary conditions change as load parameters change. High order sensitivity analysis is also performed to predict the effects of various load parameters. These formulations are based on the fact that the eigenfunctions of a freely spinning disk are orthogonal with respect to the stiffness matrix. This property may not exist for general gyroscopic continuous systems. High order sensitivity analysis of the general gyroscopic continua is more difficult and should be undertaken in the future. It is noted that the first-order sensitivity analysis performed by Chen and Bogy [8] assumes that the boundary conditions of the gyroscopic continua are independent of the load parameters, and therefore can be considered as a special case of the current analysis.

Some of the results presented in this paper can be summarized as follows.

(1) When the tangential edge load is uniform and of the follower type, the first order derivatives of the eigenvalues are real. If the direction of the edge load is opposite to the sense of disk rotation, then all the forward traveling modes will be destabilized, and all the backward and reflected waves will be stabilized. The stability property of the disk is reversed when the direction of the uniform load is reversed. The natural frequencies of all the zero-nodal-diameter modes will not be affected.

(2) When the uniform tangential edge load is conservative, the first order derivatives of the eigenvalues are zero, and the second order derivatives are purely imaginary. Reversing the direction of the edge load will not change its effects on the eigenvalues of the spinning disk. Since the first order derivatives are purely imaginary when the conservative uniform edge load is in the radial direction, we can predict that the effects of the tangential component is in general smaller than its radial counterpart.

(3) When the tangential edge load (either conservative or follower type) is of the form $f_{k} \cos k \theta$ with $k \neq 0$, the first order derivatives of all modes are zero and the second order derivatives are in general purely imaginary. Two modes will affect each other's natural frequencies only when the difference between the number of nodal diameters of these two modes is equal to $\pm k$. In the case when the non-uniform edge load is conservative it is predicted that the modal interaction of two non-reflected waves are always of the veering type. On the other hand, the modal interaction between one non-reflected wave and one reflected wave is always of the merging type. No such simple rules exist for the follower edge loads.

\section{REFERENCES}

1. J. F. Carlin, F. C. Appl, H. C. Bridwell and R. P. Dubois 1975 ASME Journal of Engineering for Industry 2, 37-48. Effects of tensioning on buckling and vibration of circular saw blades.

2. C. J. RAdCliffe and C. D. Mote JR 1977 International Journal of Mechanical Sciences 19, 567-574. Stability of stationary and rotating discs under edge load.

3. S. Chonan, Z. W. JiAng and Y. Yuki 1993 ASME Journal of Vibration and Acoustics 115, 529-534. Vibration and deflection of a silicon-wafer slicer cutting the crystal ingot.

4. J.-S. CHEN 1994 ASME Journal of Applied Mechanics 61, 788-792. Stability analysis of a spinning elastic disk under a stationary concentrated edge load. 
5. J.-S. CHEN 1996 ASME Journal of Applied Mechanics 63, 439-444. Vibration and stability of a spinning disk under a stationary distributed edge loads.

6. I. Y. SHEN and Y. Song 1996 ASME Journal of Applied Mechanics 63, 121-127. Stability and vibration of a rotating circular plate subjected to stationary in-plane edge loads.

7. K. HuSEYIN 1978 Vibrations and Stability of Multiple Parameter Systems. The Netherlands: Noordhoff International.

8. J.-S. CHEN and D. B. Bogy 1992 ASME Journal of Applied Mechanics 59, 230-235. Effects of load parameters on the natural frequencies and stability of a flexible spinning disk with a stationary load system.

9. E. ZAUDERER 1983 Partial Differential Equations of Applied Mathematics. New York: Wiley.

10. E. G. Coker and L. N. G. FILON 1957 A Treatise on Photo-Elasticity. London: Cambridge University Press.

11. G. AdAms 1987 International Journal of Mechanical Sciences 29, 525-531. Critical speeds for a flexible spinning disk. 